\title{
Editorial
}

\section{Herbst/Winter: Vitamin-D-Mangelzeit}

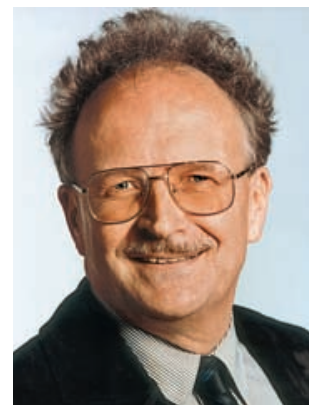

Jedes Jahr wiederholt sich dasselbe Szenario: Die Tage werden kürzer, die Nächte länger, die Sonnenstunden geringer, der endemische Vitamin-D-Mangel erfasst die Bevölkerungen der weiter vom Äquator entfernten Länder, zu denen auch Deutschland gehört. Die Unkenntnis über die Bedeutung von Vitamin D und seinen Metaboliten bei der allgemeinen Bevölkerung ist groß. Vitamin D wird ausschließlich als antirachitisches Vitamin angesehen, das man den Kleinkindern zum Skelettaufbau verabreicht. Dies ist gut so und auch die empfohlenen Dosierungen sind für Kleinkinder ausreichend. Leider wird aber die große Bandbreite der Wirkungen von Vitamin $\mathrm{D}$ beim Erwachsenen, die weit über die skelettalen Funktionen hinausgeht, nicht erkannt.

Beklagenswert ist aber nicht nur die Unkenntnis der allgemeinen Bevölkerung. Auch in Fachkreisen, die sich mit Zufuhrempfehlungen beschäftigen, werden aus den wissenschaftlichen Erkenntnissen über extraskelettale Wirkungen von Vitamin D keine Konsequenzen gezogen, sodass beispielsweise die Zufuhrempfehlungen für Erwachsene nicht weit von denen für Babies entfernt sind.

Wie bei anderen Vitaminen auch wird beim Vitamin D durch viele neuere Studien ersichtlich, dass höhere tägliche $\mathrm{Zu}$ fuhren positive gesundheitliche Wirkungen haben. Auch wenn man die Grenze zum Vitamin-D-Mangel sehr konservativ definiert als Serumkonzentrationen von 25-Hydroxy-Vitamin $\mathrm{D}_{3}$ unter $50 \mathrm{nmol} / \mathrm{l}$, so ergibt sich eine vom Mangel betroffene Zahl von mehr als einer Milliarde Menschen. Dabei zeigt sich, dass Vitamin-DMangel in den Industrieländern mit einer Vielzahl der sogenannten Zivilisationskrankheiten assoziiert ist, wie z.B. Krebs, Autoimmunerkrankungen, kardio- und zerebrovaskulären Erkrankungen, dem metabolischen Syndrom, kognitiven Störungen oder Depressionen, um nur einige zu benennen.

Polymorphismen des Vitamin-D-Rezeptorgens zeigen Assoziationen mit einer Vielzahl von Erkrankungen, wie z.B. Typ1-Diabetes und anderen Autoimmunerkrankungen, Kolon- und Prostata-Karzinom.

Vitamin-D-Mangel führt zu einer verringerten Sekretion von Insulin durch die pankreatischen Beta-Zellen, Vitamin-DGaben erhöhen nicht nur die Insulinsekretion, sondern auch die Insulinsensitivität. Eine neueste Metaanalyse unter Einschluss von ca. 100000 Personen aus 28 Studien zeigt, dass eine verbesserte Versorgung mit Vitamin D (gemessen an den 25-Hydroxy-Vitamin- $\mathrm{D}_{3}$-Spiegeln im Serum) mit einem drastisch reduzierten Risiko kardiometabolischer Erkrankungen wie z.B. dem metabolischen Syndrom und Typ-2-Diabetes assoziiert ist (Maturitas 2010; 65: 225-236).

Ähnliche Beziehungen konnten auch zwischen der Vitamin-D-Versorgung und der Inzidenz von Depressionen gefunden werden, die nicht überraschend in der Vitamin-D-armen dunklen Jahreszeit massiv zunehmen (Circulation 2009; 120: S451).

Vor diesem Hintergrund sind die derzeitigen Zufuhrempfehlungen für Vitamin D als äußerst fragwürdig zu bezeichnen. Kaum erträglich sind darüber hinaus die in letzter Zeit gehäuften medialen Attacken gegen eine Verbesserung der Vitaminversorgung etwa unter dem Stichwort „Vitaminlüge“. Daher im Interesse gesundheitlicher Prävention: In der dunklen Jahreszeit eine Zufuhr von 2000 IU Vitamin $D$ pro Tag sicherstellen und gelegentlich zur Sicherheit 25-OH-Vitamin $\mathrm{D}_{3}$ im Serum messen.

Prof. Dr. Dr. Karlheinz Schmidt 